
allemande

51-2| 2019

Les Humanités environnementales : circulations et renouvellement des savoirs en France et en Allemagne

\title{
Sasha DISKo, The Devil's Wheels. Men and Motorcycling in the Weimar Republic
}

New York/Oxford, Berghahn Books, 2016

Louise-Hélène Filion

\section{OpenEdition}

Édition électronique

URL : https://journals.openedition.org/allemagne/2064

DOI : 10.4000 /allemagne.2064

ISSN : 2605-7913

Éditeur

Société d'études allemandes

Édition imprimée

Date de publication : 10 décembre 2019

Pagination : $527-529$

ISSN : 0035-0974

Référence électronique

Louise-Hélène Filion, "Sasha disko, The Devil's Wheels. Men and Motorcycling in the Weimar Republic », Revue d'Allemagne et des pays de langue allemande [En ligne], 51-2 | 2019, mis en ligne le 02 décembre 2019, consulté le 21 mai 2021. URL : http://journals.openedition.org/allemagne/2064 ; DOI : https:// doi.org/10.4000/allemagne.2064 


\section{Italiques}

Sasha DIsko, The Devil's Wheels. Men and Motorcycling in the Weimar Republic, New York/Oxford, Berghahn Books, 2016, 360 p.

Dans une entreprise qui, bien qu'elle demeure ancrée dans la démarche historienne, rappelle parfois celle de Kristin Ross dans son fameux ouvrage Fast Cars, Clean Bodies ${ }^{(1)}$, Sasha Disko propose que la motocyclette a fait figure d'objet médiateur essentiel pour l'accès à la modernité d'un grand nombre d'Allemands, pendant la période de Weimar - une modernité qu'il faut ici avant tout comprendre comme une expérience de la mobilité (une mobilité spatiale concrète dans les loisirs, par exemple, mais aussi sur le plan des classes sociales, sur le plan sexuel, etc.). À la croisée de l'histoire de la motorisation, de l'histoire de la consommation et d'une histoire culturelle prenant fortement appui sur des études de genre, son travail se réclame précisément des recherches interdisciplinaires récentes dans le champ des Mobility Studies, et notamment de l'ouvrage de Gijs Mom, Atlantic Automobilism. Emergence and Persistence of the Car ${ }^{(2)}$. Disko se situe donc dans la droite ligne de travaux qui ont envisagé les transports en fonction des enjeux sociaux et politiques qu'ils soulèvent, tout en renouvelant, par le choix même de son objet d'étude, certaines voies ouvertes par ces travaux qui se sont le plus souvent attachés à l'automobile, cette marchandise «par excellence» du Xxe siècle. Un autre point central de sa démarche consiste à poser l'hypothèse, pour la période de Weimar, de la construction d'identités masculines concrètes, modernes et marquées par leur hétérogénéité, à travers la consommation - envisagée ici par le biais précis de la motocyclette -, alors que, pour cette période, on sait l'importance que les historiens ont accordée aux représentations de la consommation féminine. L'ouvrage, qui se déploie sur sept chapitres, est ambitieux par la diversité des documents d'archives et des sources qu'il convoque: les archives officielles, politiques et légales, ont permis notamment d'établir les statistiques relatives à la possession d'une moto, mais

1 Kristin Ross, Fast Cars. Clean Bodies. Decolonization and the Reordering of French Culture, Cambridge, MIT Press, 1995.

2 Gijs Mom, Atlantic Automobilism. Emergence and Persistence of the Car, 1895-1940, New York/ Oxford, Berghahn (coll. «Explorations in Mobility»), 2015. 
aussi de présenter les lois relatives à l'utilisation de ce véhicule pendant la période de Weimar; les archives des clubs consacrés à la conduite, celles des fameux ADAC (Allgemeiner Deutscher Automobil-Club) et DMV (Deutscher Motorradfahrer-Verband), et les archives des fabricants de motos les plus importants de l'époque; les revues destinées aux amateurs de la conduite motorisée, qui prolifèrent pendant la période de Weimar; les textes de fiction consacrés à la moto et publiés en tant que nouvelles, poèmes, ou romans-feuilleton dans les revues et magazines dédiés à la motorisation, etc. Le dépouillement des sources industrielles et officielles permet par exemple à l'auteure d'illustrer que, au-delà du contexte économique de l'après-Grande Guerre dont le déséquilibre a certes en très grande partie contribué à la prédominance de la motocyclette, la rationalisation de la production et les programmes incitatifs mis en place par l'État ont aussi contribué à la démocratisation de l'accès à la motocyclette (p. 60). En définitive, c'est toutefois l'interprétation des débats menés dans les revues - par des lettres à l'éditeur, notamment - ainsi que celle des textes fictionnels, qui fondent la richesse de la démarche analytique de l'auteure. De ce point de vue, les deux derniers chapitres de l'ouvrage mettent en valeur des sources fascinantes, restituant l'existence de femmes motocyclistes et/ou auteures engagées au sein du discours majoritairement masculin sur la moto et du milieu de la course motorisée, et réfléchissant aux impacts de la motocyclette sur les relations entre les sexes. Dans l'avant-dernier et sixième chapitre ("Motoring Amazons? Women and Motorcycling») qui expose bien comment, en dépit de nombreux obstacles, certaines femmes ont activement embrassé le rapport à la technologie pendant la période de Weimar, en battant même des hommes dans les courses de motocyclettes tout en étant parfois les seules à terminer la course (p. 257), s'imposent ainsi les articles d'Alfred Nauck; ingénieur, auteur de plusieurs ouvrages techniques, Nauck avait même imaginé que la femme motocycliste pourrait avoir un passager homme (p. 268-269). Dans le dernier et septième chapitre ("Sex and the Sidecar. Sexuality, Courtship, Marriage, and Motorization»), l'analyse de la nouvelle Die Motorbraut de Georg Oswald Breyer est captivante; elle éclaire très bien le renversement des rôles traditionnels associés à la masculinité et à la féminité bourgeoises que décrit Breyer; ainsi, dans cette nouvelle, la passion de l'épouse pour la moto est si vive et prenante que, jusqu'au premier jour des leçons, elle transforme le bureau de son mari - espace privé masculin s'il en est - en atelier pour confectionner ses vêtements de sport, et contraint son mari à prendre ses repas à la taverne (p. 308).

Si l'hypothèse principale du lien entre la motocyclette et l'expérience de la modernité convainc - on soulignera d'ailleurs le souci de Disko d'identifier les comparaisons établies par les acteurs du discours allemand sur la moto avec d'autres modèles modernes, a fortiori le modèle américain et sa moralité parfois jugée prude -, le choix de postuler le caractère primordial de la construction d'identités masculines à travers la motocyclette ne suscitera peut-être pas l'adhésion aussi marquée du lecteur, possiblement en raison de la division du plan. Ainsi, dans les deux derniers chapitres, l'analyse des documents que l'on a mentionnés fait en sorte qu'en fin de parcours, l'ouvrage accorde aussi une place essentielle aux constructions de la féminité, dont les savoureuses représentations fictionnelles sont adroitement mises en valeur. Pourtant, en dépit du caractère parfois quelque peu répétitif de l'argumentation - notamment au chapitre 5, lorsqu'il est question des principaux comportements déviants qui ont mené à l'élaboration de stratégies destinées à éluder les préjudices sociaux entraînés par l'augmentation notable du nombre de motocyclettes sur les routes -, Disko présente d'adéquate manière les types de masculinité «idéale» associés aux motocyclistes pendant la période de Weimar (chapitre 3), ainsi que les divergences d'opinion, aussi bien au sein de la communauté des 
motocyclistes, que dans la République de Weimar au sens large, sur les comportements qui correspondraient à une masculinité "convenable». Souvent, elle relie pertinemment ces différences d'opinion à divers enjeux centraux qui suscitent le débat pendant la période: les débats sur la nature du «sport», et sur la possibilité ou non d'associer la motocyclette à un sport, sont savamment étudiés au chapitre 4. Toutefois, il arrive que les conclusions générales tirées à la fin d'un chapitre ou d'une section surprennent, l'auteure terminant par un propos très affirmatif et/ou généralisant, sans que les analyses menées paraissent justifier l'aspect catégorique de la conclusion avancée - c'est rarement le cas, certes, mais l'on se doit néanmoins de signaler la présence de conclusions de ce type, au chapitre 5 par exemple: "The process of motorization, particularly rife with both contradictions and the potential for sparking social conflict during the Weimar Republic, heightened the sense that intense instability and crisis were perpetual. Motorcyclists appeared to embody the disorder of spatial and social relations" (p. 242). Outre les chapitres déjà évoqués, notons que les deux premiers chapitres sont consacrés respectivement à la croissance de l'industrie allemande de la motocyclette pendant ses 40 premières années (chapitre 1), à la figure de l'ingénieur et à son rôle dans les discussions qui cherchent à réfléchir à l'articulation entre la technologie et la culture, ainsi qu'à tout ce qui concerne l'univers publicitaire entourant la motocyclette (chapitre 2). En dépit des quelques réserves émises, l'ouvrage contient des aperçus fascinants sur les principaux débats et polémiques sociaux qui ont entouré la gestion des loisirs et la transformation de l'espace routier, pendant cette période qui voit l'Allemagne devenir le chef de file mondial de la production de motocyclettes.

Louise-Hélène Filion

Marion ABALLÉA, Un exercice de diplomatie chez l'ennemi. L'Ambassade de France à Berlin, 1871-1933, Villeneuve d'Asca, Presses universitaires du Septentrion, $2017,422 p$.

L'ouvrage de Marion Aballéa, issu d'une thèse de doctorat en cotutelle (Strasbourg) Genève), constitue une contribution importante au renouvellement de l'histoire des relations internationales. L'auteure a pris pour objet ce qui, au premier regard, pourrait paraître comme un élément de l'histoire diplomatique traditionnelle, mais loin de faire une histoire de plus des relations diplomatiques franco-allemandes, elle mène une "histoire totale» d'une ambassade considérée dans son ancrage local - ici Berlin.

Après avoir défini l'"ambassade» - en tant qu'institution, avec ses mécanismes déterminant des pratiques, animée par des centaines d'individus occupant les échelons les plus variés et ayant des missions diverses -, elle procède à une socio-histoire de ce microcosme français à Berlin et multiplie les perspectives portées sur son objet: histoire sociale et culturelle, histoire des pratiques diplomatiques et histoire du symbolique appliquée à la diplomatie. Ainsi, un magnifique chapitre 7 ("l'ambassade mondaine») est consacré notamment à l'architecture du bâtiment, à la décoration intérieure, à la symbolique des réceptions officielles, fondée notamment sur le décryptage des menus. Elle se livre aussi à une très convaincante analyse prosopographique du personnel (chapitre 5) diplomatique et non diplomatique de l'ambassade (allant jusqu'aux agents du chiffre, aux cuisiniers, au personnel de maison), retraçant des "parcours» de diplomates et le rapport entre diplomates, personnel français et étranger. Elle entend ainsi retrouver les traces du quotidien de l'ambassade, de son 